\title{
Differential expression of invasion promoting genes in childhood rhabdomyosarcoma
}

\author{
SORIN ARMEANU-EBINGER ${ }^{1}$, MICHAEL BONIN ${ }^{2}$, KARINA HÄBIG $^{2}$, CHRISTOPHER POREMBA $^{3}$, \\ EWA KOSCIELNIAK ${ }^{4}$, JAN GODZINSKI ${ }^{5,6}$, STEVEN W. WARMANN ${ }^{1}$, JÖRG FUCHS $^{1}$ and GUIDO SEITZ ${ }^{1}$ \\ ${ }^{1}$ Department of Pediatric Surgery, University Children's Hospital; ${ }^{2}$ Institute of Anthropology and Human Genetics, \\ Microarray Facility, University Hospital, Tübingen; ${ }^{3}$ Department of Pathology, University Hospital, Düsseldorf, Germany, \\ now Center of Histopathology, Cytology and Molecular Diagnostics, Trier; ${ }^{4}$ Klinikum Stuttgart, Department of Pediatric \\ Oncology/Hematology, Olgahospital Stuttgart, Germany; ${ }^{5}$ Department of Pediatric Surgery, Marciniak Hospital, \\ Wroclaw; ${ }^{6}$ Department of Surgical Oncology for Children and Adolescent, Mother and Child Institute, Warsaw, Poland
}

Received October 19, 2010; Accepted December 13, 2010

DOI: $10.3892 /$ ijo.2011.921

\begin{abstract}
Expression profiling of tumor tissue allows a systematic search for targeted therapies and offers relevant prognostic information. Molecular studies on rhabdomyosarcoma (RMS) revealed a more differentiated classification than the histological subgrouping into embryonal (RME) and alveolar (RMA) rhabdomyosarcoma, and reflected the chromosomal aberrations found in RMS. We addressed biological processes like cell migration and emerging drug resistance by expression profiling to identify mechanisms of metastasic invasion and differential response to chemotherapy in RMS. Gene expression analysis was performed in 19 RMS samples using the Affymetrix U133 Plus2 array. Validation of target genes was performed by qRT-PCR. Data were analyzed using Pathway analysis software. Involvement of these genes in invasion processes was evaluated in knock-down experiments using specific interference RNA and Matrigel ${ }^{\mathrm{TM}}$ invasion assay. In RMA tissues 211 of 534 genes were overexpressed, in RME tissues 323 genes were overexpressed. Pathway analysis software identified a group of genes involved in cell growth, morphology and motility. In patients with distant metastases especially transcription factors such as FOXF1 and LMO4 showed a high expression, which were described as determinants of tumor cell migration. Down-regulation of these factors inhibited the invasion of RMS cells $>10$-fold. Microarray technology is a powerful method not only to classify RMS samples, but also to identify major regulatory processes. Functional evaluation of LMO4 and FOXF1 identified targets of a molecular network for preventing metastatic invasion in RMS.
\end{abstract}

Correspondence to: Dr Sorin Armeanu-Ebinger, Department of Pediatric Surgery, University Children's Hospital, Hoppe-SeylerStrasse 1, 72076 Tübingen, Germany

E-mail: sorin.armeanu-ebinger@med.uni-tuebingen.de

Key words: rhabdomyosarcoma, gene expression, invasion, microarray, knock-down

\section{Introduction}

Rhabdomyosarcoma (RMS) is the most common pediatric soft tissue sarcoma. About two-thirds of all sarcomas and $7-8 \%$ of all malignant solid tumors in childhood are RMS (1). The two main histological subtypes are embryonal (RME) and alveolar (RMA) RMS. The initial prognosis of the patients has been related to the primary tumor localization, histological subtype, stage of disease, and the age at diagnosis $(2,3)$. Histological evaluation has been improved by a combination of the four biomarkers like epidermal growth factor receptor (EGFR), fibrillin-2, AP2 $\beta$, and P-cadherin. These biomarkers allow a better differentiation between RME and RMA and have been implemented in the routine diagnosis of RMS (4). Genetic subtyping has identified PAX3-FKHR-fusion positive, PAX7-FKHR-fusion positive, and fusion-negative subsets of RMA; the clinical significance of these subsets is still being actively investigated $(5,6)$. High output methods such as DNA microarrays, proteomics and tissue-based arrays revealed genotypic and phenotypic signatures which may be useful for a profound diagnostic, prognostic and predictive purpose in the highly heterogenic RMS. So far, molecular classification of RMS is performed by gene expression analysis (6-13), single nucleotide polymorphism analysis for $\mathrm{LOH}$ determination (7), and comparative genomic hybridization (8). The genetic classification of RMS revealed a group of RMA with high similarity to RME, suggesting a more appropriate definition of tumors using histological appearance and genetic markers. RMA samples without the characteristic chromosomal translocation $\mathrm{t}(2 ; 13)$, PAX3/FKHR, PAX7/FKHR were similar to RME when gene expression and LOH pattern were compared (7). This genomic analysis can be conducted also for detecting targets for specific therapy of RMS entities. Expression profiling of PAX3/FKHR-specific gene signatures served to identify physiologically important target genes to understand pathophysiological functions and develop therapeutic agents for treatment of RMA $(9,14,15)$. Deregulation of other signal transduction pathways were detected focusing on drug development involving tyrosine and serine/threonine kinases 
Table I. Patient data.

\begin{tabular}{lcccc}
\hline Array no. & Diagnosis & Age $(\mathrm{y})$ & Tumor localisation & Metastases \\
\hline I5R_032a01 & RMA & 9 & Perianal & Yes \\
I5R_032a06 & RME & 5 & Prostata/pelvis & Yes \\
I5R_032a07 & RMA & 12 & Forehand & Yes \\
I5R_032a08 & RMA & 7 & Bladder & Yes \\
I5R_032a09 & RME & 2 & Bladder/prostate & No \\
I5R_032a10 & RME & 3 & Bladder/prostate & No \\
I5R_032a12 & RME & 2 & Pelvis & No \\
I5R_032a13 & RME & 8 & Diaphragm & No \\
I6R_002a01 & RME & 1 & Abdomen & Yes \\
I6R_002a03 & RMA & 2 & Thigh & Yes \\
I6R002a05 & RME & 11 & Forearm & No \\
I6R_002a06 & RMA & Forearm & Yes \\
I6R_002b01 & RMA & 15 & Foot & Yes \\
I6R_002c02 & RMA & 15 & Pelvis & Yes \\
I6R_002c03 & RME & 9 & Thoracic & No \\
I6R_002c04 & RMA & 3 & Abdomen & No \\
I6R_002c05 & RME & 0 & Abdomen & No \\
I6R_002c06 & RME & RME & Diaphragm & Yes \\
I6R_002c07 & & & No \\
\hline
\end{tabular}

with another high throughput method using tissue microarray slides. mTOR and PKC pathways seem to be activated in RMS $(16,17)$. Expression profiles allow a systematical analysis of specific biological processes like cell migration and emerging drug resistance to address mechanisms of metastatic invasion and differential response to chemotherapy and radiotherapy (18). Therefore, we performed gene expression analysis of RMS tumor samples and knock-down experiments with siRNA in RMS cells to identify and evaluate the role of genes involved in the invasion of RMS cells.

\section{Material and methods}

Tumor specimens. All patients were included and treated within the trials of the cooperative soft tissue sarcoma study (CWS) Group of the Society of Pediatric Oncology and Hematology (GPOH). One patient was treated within the CWS 96 trial and 18 patients were treated within the CWS 2002-P trial. Of these 19 samples, 5 tumor samples were allocated by the CWS study group from Stuttgart. Nine samples were obtained from our own institution and 5 samples were provided by the Department of Pathology from the University of Düsseldorf. Patient data are shown in Table I. Mean age of patients was 6.4 years (range 1-15 years, 95\% CI of mean 4.2-8.8). Histological analysis revealed 8 RMA and 11 RME based on the International classification of rhabdomyosarcoma criteria. All cases received central pathological review and patients were treated according to the uniform protocols. Only samples with tumor cell content of at least $80 \%$ were included for analysis. Metastases were present at the time of surgery in $7 / 8$ patients suffering from RMA and in 3/11 with diagnosis of RME. The studies were approved by the local ethics committees (CWS 96: EK LÄK 105/95, CWS 2002-P: 418/2004V).

RNA extraction and oligonucleotide microarrays. Tumor specimen were immediately fixed in liquid nitrogen and stored at $-80^{\circ} \mathrm{C}$ for further gene chip analysis. Therefore, total RNA extraction was carried out using RNeasy Kit (Qiagen, Hilden, Germany). The quality of total RNA of tumors was monitored by Agilent 2100 Bioanalyzer using the RNA 6000 Nano LabChip Kit (Agilent Technologies, Boeblingen, Germany) as specified by the manufacturer. Samples with RIN $>8$ were considered for gene chip analysis.

Affymetrix high-density oligonucleotide microarrays (GeneChip ${ }^{\circledR}$ Human Genome U133 Plus 2.0 Array, Affymetrix, Santa Clara, CA, USA) were used for gene expression analysis. Hybridization experiments and evaluation was done by the Microarray Facility Tübingen. Arrays were scanned at 3- $\mu \mathrm{m}$ resolution using a GeneChip System confocal scanner (Agilent Technologies). Scanned images were subject to visual inspection and analyzed using the Affymetrix's Microarray Suite version 5.0 (MAS 5.0) algorithms to generate report files for quality control. We standardized the data for variations in staining by scaling the average of the fluorescence intensities of all genes on an array to constant target intensity for all arrays used (150). The signal intensity for each gene was calculated as the average intensity difference, represented by $\Sigma[(\mathrm{PM}-\mathrm{MM}) /($ number of probe pairs) $]$, where PM and MM 
Table II. siRNA and oligonucleotides for qRT-PCR.

\begin{tabular}{lllll}
\hline Transcript & siRNA (Qiagen) & \multicolumn{1}{c}{ Forward sequence 5'-3' } & \multicolumn{2}{c}{ Reverse sequence 5'-3' } \\
\hline LMO4 & SI03185777 & AAAGTGGCATGATCCTTTGC & ACGAGTTCACTCGCAGGAAT & 100 \\
FOXF1 & SI00420833 & CGTATCTGCACCAGAACAGC & TGGCGTTGAAAGAGAAGACA & 117 \\
PDHB & & GGTTTCCCATTCAAGACCTG & TGGTTTCCATGTCCATTGGT & 119 \\
control siRNA & \multirow{2}{*}{1022076} & Non-silencing & & \\
\hline
\end{tabular}

denote perfect match and mismatch probes. In the samples, the mRNA of a gene was considered expressed (present) when the detection $\mathrm{p}$-value and change $\mathrm{p}$-value were $<0.05$. To determine the p-values, a signed rank analysis was carried out on the PM and MM differences comparing each probe pair. The resulting p-values were used to make the change calls. Genes with significantly varying expression in RMA and RME were identified using the Data Mining Tool (Affymetrix). Biological mechanisms, pathways and functions of the selected genes were identified by Ingenuity Pathways Analysis. The data sets containing gene identifiers and corresponding expression change values from the previously selected 534 genes were mapped to its corresponding gene object in the ingenuity pathways knowledge base. These genes, called focus genes, were overlaid onto a global molecular network developed from information contained in the ingenuity pathways knowledge base. Networks of these focus genes were then algorithmically generated based on their connectivity. Canonical pathway analysis identified the pathways from the Ingenuity Pathway Analysis library that were most significantly associated to the data set. The significance of the association between the data set and the canonical pathways was measured by Fischer's exact test. The calculated p-value determined the probability that the association between the genes in the dataset and the canonical pathway is explained by chance alone.

The statistical technique SAM (significance analysis of microarrays) was used for finding significant genes in the set of microarray experiments whereas two class unpaired data (metastasized vs. not metastasized tumors) were compared by 5000 permutations and false discovery rate of $0.7 \%$. Genes positively regulated $(n=157)$ and negatively regulated $(n=46)$ with a score $(d)>2$ were tested for a significant regulation (ANOVA test, $\mathrm{p}<0.05$ ) among the two groups of samples (metastasized vs. not metastasized) and were hierarchically clustered by Euclidian distance using the Expression Profiler at the EBI (19). For functional analysis of analyzed genes possibly responsible for metastatic invasion, siRNA-knock down and invasion assays were carried out.

Cell culture experiments. The embryonal rhabdomyosarcoma cell line RD (ATCC, Manassas, VA, USA) and the alveolar rhabdomyosarcoma cell line Rh30 expressing the Pax3/FKHR fusion protein secondary to the $\mathrm{t}(2 ; 13)(\mathrm{q} 35 ; \mathrm{q} 14)$ translocation (DSMZ, Braunschweig, Germany) were cultured in DMEM medium supplemented with $10 \%$ fetal calf serum, $4.5 \%$ L-Glu and $2.5 \%$ HEPES in a humified atmosphere containing $5 \%$ $\mathrm{CO}_{2}$ at $37^{\circ} \mathrm{C}$. All cell cultures were mycoplasma species negative and were tested for contamination and integrity using the PowerPlex 16 Assay (Promega, Mannheim, Germany). The analysis was done in the Institute for Human Genetics, Tübingen, in May 2010.

Transfection. Embryonal and alveolar rhabdomyosarcoma cells $\left(5 \times 10^{4}\right.$ cells) were seeded out in 24 -well plates (FalconBecton Dickinson Labware, Franklin Lakes, NJ, USA) and were cultured as described above. At day two, transfection was carried out with $3 \mu \mathrm{l}$ siRNA $(5 \mathrm{nM})$ (Table II) and $3 \mu \mathrm{l}$ HiPerFect (Qiagen) for each cell line and siRNA in triplicates. One control transfection was performed with non-silencing control siRNA-AF488 and one without siRNA. Two days later, cells were recovered by trypsin treatment and used for migration assays and RNA isolation.

Invasion assay. For invasion assays BD BioCoat ${ }^{\mathrm{TM}}$ Growth Factor Reduced (GFR) Matrigel Invasion Chambers were used as recommended by BD Biosciences. Transfected cells $\left(5 \times 10^{4}\right)$ were overlaid on the reconstituted Matrigel in triplicates for each transfection experiment and cultured for $22 \mathrm{~h}$. Cells in the upper chamber were removed by scrapping. Migrated cells were fixed with $4 \%$ buffered paraformaldehyde, stained with H33342 and counted using a fluorescence microscope. Migration index was calculated as percentage of migrated cells related to total cells in the assay. Relative migration represents migration index compared to control cultures transfected with non-sense siRNA.

$R N A$ isolation, $c D N A$ preparation and quantitative real-time $P C R$ ( $q R T-P C R)$. Total RNA of cells ( $48 \mathrm{~h}$ after RNAi treatment, and before invasion experiments) was isolated using the RNeasy Micro Kit (Qiagen) including a DNase digest on the column with RNase-Free DNase Set (Qiagen). The RNA quality (RIN>8) was controlled by Lab-on-Chip-System Bioanalyser 2100 (Agilent) and the amount of isolated RNA was determined using a Nanodrop ${ }^{\circledR}$ photometer (Peqlab, Erlangen, Germany). cDNA synthesis was performed with $1 \mu \mathrm{g}$ of total RNA using the QuantiTect Reverse Transcription Kit (Qiagen). For examination of genomic DNA contamination, we included a reverse transcription-negative control. The qRT-PCR and relative quantification was done as described by Häbig et al, except of using $2 \mu \mathrm{l}$ of 1:10 diluted cDNA in $10 \mu \mathrm{l}$ reaction volume (20). For quantification of mRNA of LMO4 and FOXF1 the listed exon-exon boundary spanning oligonucleotides were applied (Table II). PDHB was used as a reference gene.

Statistical analysis. Statistical analysis between the groups was carried out using one way ANOVA on ranks test and 


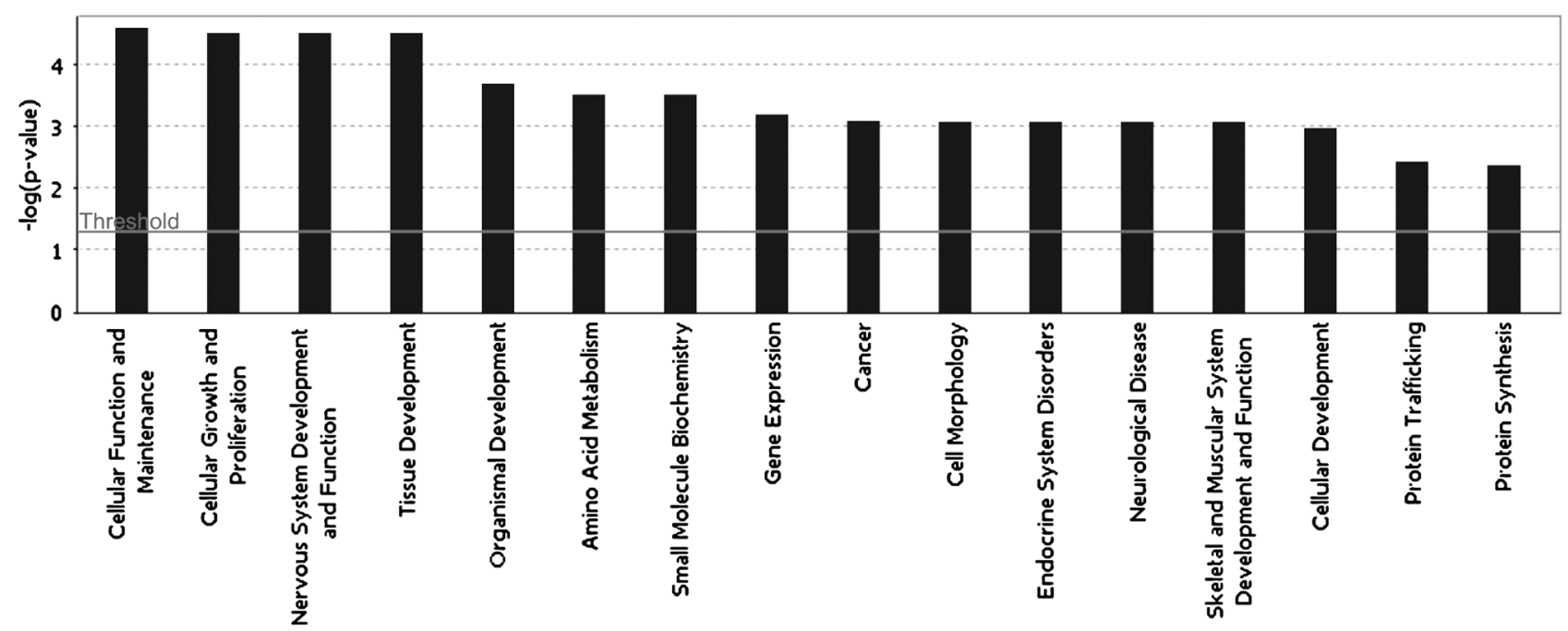

Figure 1. Functional analysis of genes differentially expressed in RMA and RME. Genes from the dataset that were changed $>2$-fold (cut-off of $p<0.002$ ) were considered for the analysis and were associated with biological functions and diseases in the ingenuity pathways knowledge base. Functional analysis identified the biological functions and/or diseases that were most significant to the data set.
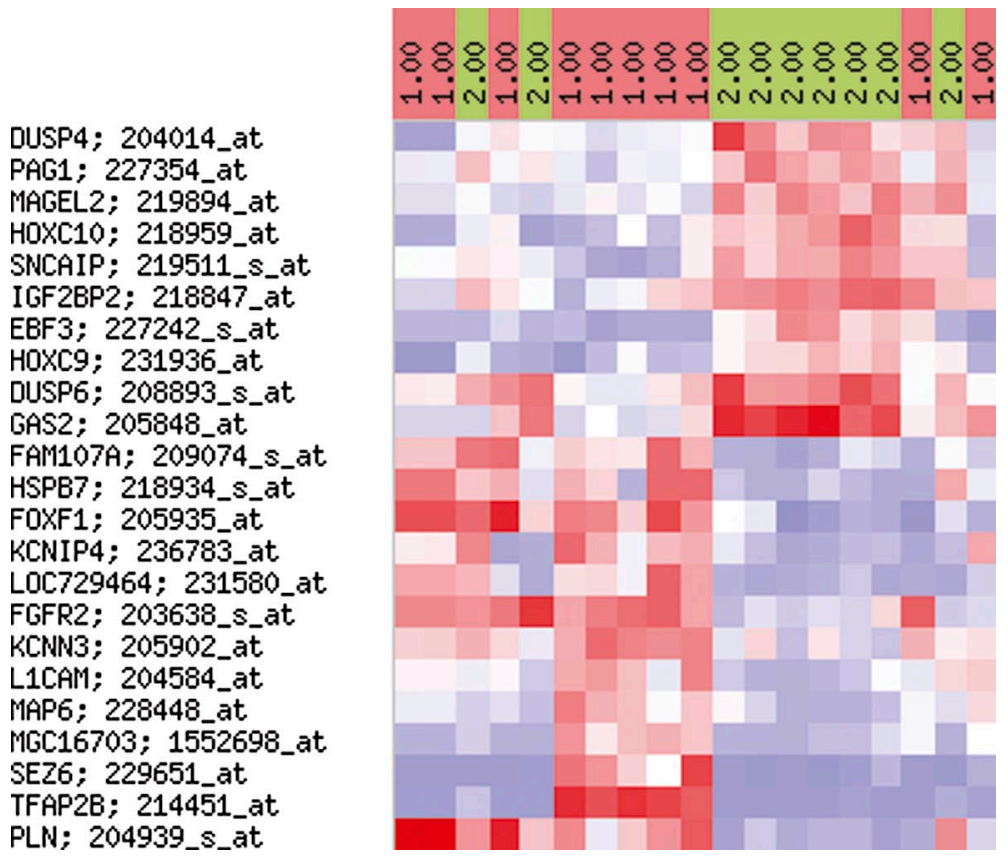

Figure 2. Hierarchical clustering of differentially expressed genes in RMS tumors with metastases (1) and without metastases (2). The color of the boxes varies from enhanced expression (dark red) to decreased expression (dark blue) in tumors from patients with metastases.

GraphPad Prism 4.00 (GraphPad Software). All numeric data are expressed as the mean \pm SD. Significance was assumed for all results with $\mathrm{p}<0.05$.

\section{Results}

Differentially expressed genes in RMA and RME. In order to examine the biological pathways involved in RMS, we analyzed the expression profile of 8 RMA and 11 RME specimens. Statistical analysis indicated 534 differentially expressed genes, of which 211 genes were prevalently expressed in RMA tissues. Transcripts from 323 genes were significantly over-expressed in RME ( $\mathrm{p}<0.005$ and fold-change $>2$ ). Comparative analysis of biological functions of differentially expressed transcripts in RMA and RME was carried out using the ingenuity pathways analysis (IPA) software. Biological mechanisms, pathways and functions of the selected genes were compared. The most probable biological pathways including the 534 selected genes from the differential expression analysis of RMA and RME samples are given in Fig. 1. Most of the differentially expressed genes were related to functions regarding tissue development processes in general, of the nervous system and of cells in particular. Proliferation and biochemical pathways like amino-acid metabolism, protein synthesis and trafficking 


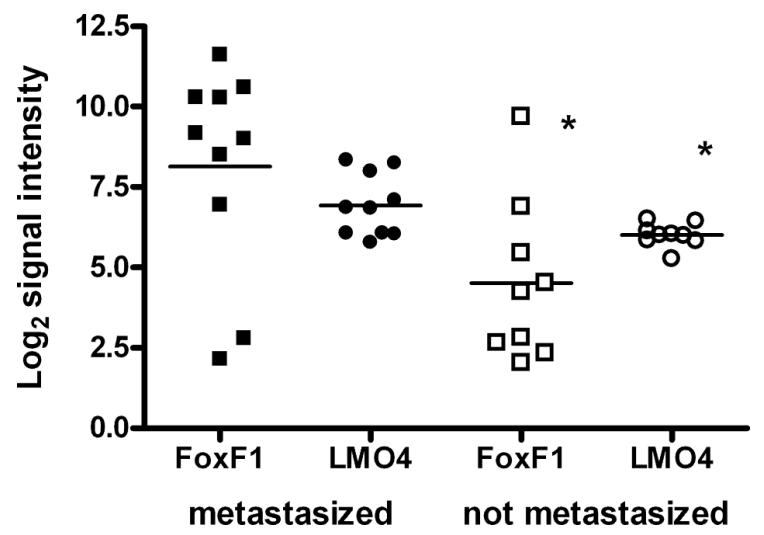

Figure 3. Expression of selected migration factors in RMS tissue samples. Gene expression of LMO4 and FOXF1 was detected in metastasized and non-metastasized tissue using the microarray expression analysis. Relative signal intensity of the probe set for LMO4 and FOXF1 for each tissue sample are shown. Signal means for each gene are significantly different when the groups of tumors with metastases (black symbols) and without metastases (open symbols) were compared in an unpaired t-test $(" \mathrm{p}<0.05)$

have been found significantly associated with the 534 probe set. In the group of genes involved in cell growth and cell morphology, we identified cell motility-associated transcription factors FOXF1 and LMO4 with higher expression in RMA than in RME (19.2- and 1.9-fold, respectively). This differential regulation of important pathways in RMA and RME may reflect tumor characteristics such as cell proliferation and metastatic invasion. Consecutively, we evaluated the expression profile based on patient data regarding metastases.

Metastatic invasion-associated genes were filtered within the sample pool by significance analysis of microarrays. Two groups of samples (metastasized vs. not metastasized) were compared. From the set of 534 genes we selected genes with higher $(n=157)$ and lower $(n=46)$ expression in RMA compared to RME. Supervised hierarchical clustering by Euclidian distance using the Expression Profiler at the EBI identified two clusters of genes differentiating tumors based on associated metastatic patterns (Fig. 2). This revealed the homogeneity of tumor samples from patients with metastases independent from the histological classification into RMA and RME subtypes.
Role of LMO4 and FOXF1 in the invasiveness of RMS cells. Among genes differentially expressed in the patient groups with or without distant metastases, we identified FOXF1 and LMO4 which are coding for transcription factors described as determinants of tumor cell migration $(21,22)$. LMO4 transcripts were detected in both, metastatic $(\mathrm{n}=11)$ and non-metastatic $(\mathrm{n}=8)$ RMS samples with a significant 2.4-fold higher expression in RMA (unpaired t-test, $\mathrm{p}=0.0026$, Fig. 3). FOXF1 was detected in the same analysis as highly expressed in metastatic tumor samples and with a significant lower expression in non-metastatic samples (unpaired t-test, $\mathrm{p}=0.015$, Fig. 3). As these experiments indicated a differential expression of migration factors in RMS tumors, we performed expression and functional analysis of LMO4 and FOXF1 with corresponding tumor cell lines: Rh30 for RMA and RD for RME.

The mRNA for LMO4 and FOXF1 in RMS cell lines was quantified by qRT-PCR. LMO4 was detected in Rh30 cells at levels 3.6 times higher than in embryonal RD cells. The relative quantification of FOXF1 revealed a 5-times higher expression in Rh30 cells compared to RD cells, which reflects the expression pattern observed in primary tumor samples (Fig. 4A). For functional analysis of the migration factors they were knocked down by means of RNA interference. The level of mRNA for both LMO4 and FOXF1 was suppressed in both RMS cell types upon transfection with the corresponding specific siRNA down to $25 \%$ of the transcripts in control cultures transfected with nonsense siRNA (Fig. 4B).

Invasiveness of the RMS cells was monitored in an invasion chamber using Matrigel as matrix and $10 \%$ FCS as chemoattractant in the lower chamber. Up to $76 \%$ of the Rh30 cells migrate in the $22 \mathrm{~h}$ of the invasion assay through the Matrigel layer. In the same period only $43 \%$ of the RD cells reached the lower chamber (Fig. 5A). RMS cells transfected with siRNA specific for LOM4 and FOXF1 revealed a lower invasion index in the same migration experiments. The relative migration was 10 times lower in Rh30 cells transfected with LMO4 and FOXF1 siRNA compared to control cultures transfected with non-sense siRNA (Fig. 5). Knocking down LMO4 in RD cells lead to $92 \%$ inhibition of cell invasion compared to the control cells. Similar experiments targeting FOXF1 did not significantly change the invasion characteristics of RD cells.
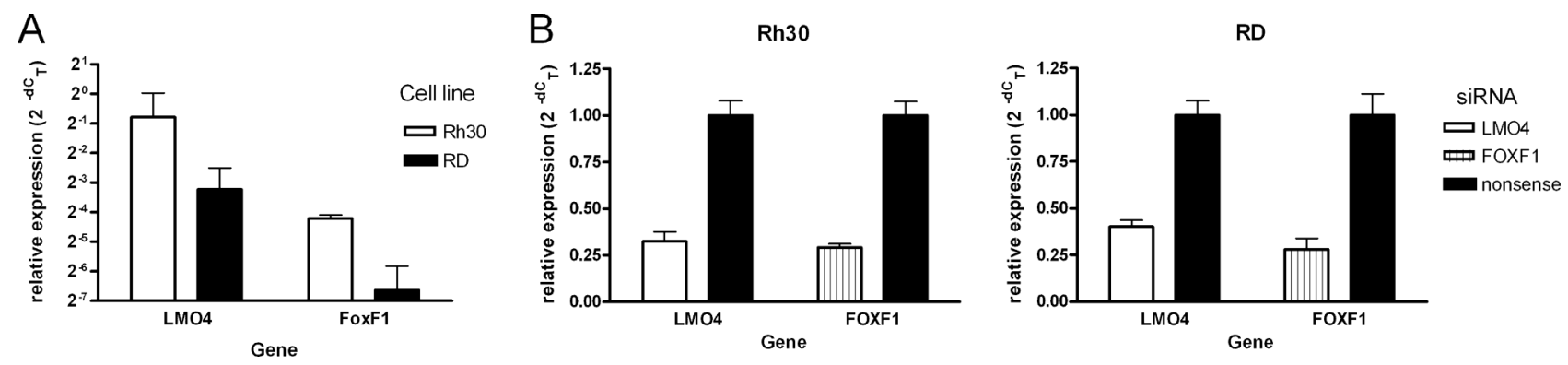

Figure 4. Reduction of LMO4 and of FOXF1 gene expression with specific siRNA. (A) Abundance of mRNA coding for LMO4, FOXF1 and of the control gene PDH was quantified in alveolar (Rh30) and embryonal (RD) RMS cells by qRT-PCR. Relative expression was calculated using PDHB as house-keeping gene. Both cell lines expressed LMO4 and FoxF1. Data are the means and SD from 3 experiments. (B) RMS cells were transfected with indicated siRNA and cultured for $48 \mathrm{~h}$. Expression of LMO4 and FOXF1 was quantified by qRT-PCR using the control gene PDHB as house-keeping gene and transfection control with non-sense siRNA. Data are means and SD from 4 experiments. 
A

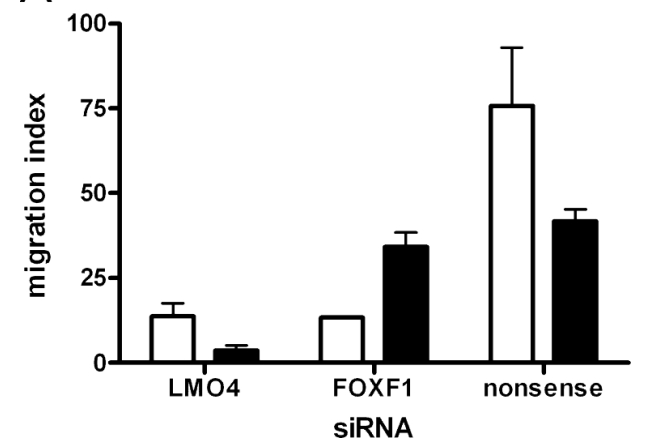

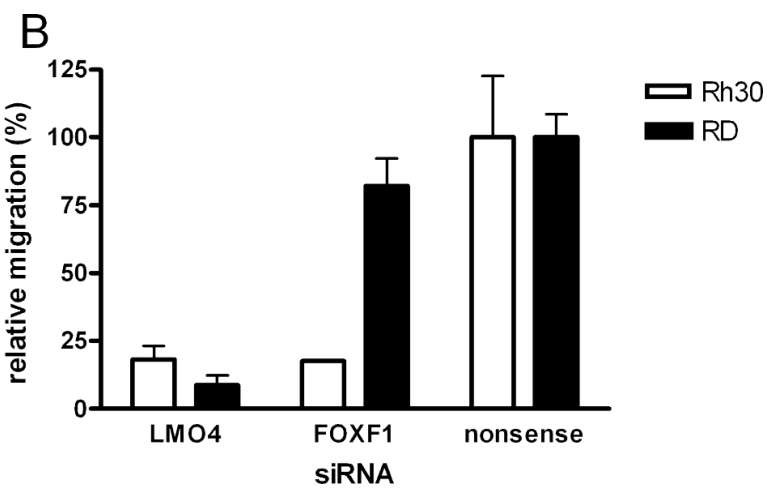

Figure 5. Blocking of cell migration by siRNA specific for LMO4 and FOXF1. RMS cells were transfected with the indicated siRNA and cultured for $48 \mathrm{~h}$. Transfected cells were placed on Matrigel in a migration assay for $22 \mathrm{~h}$. Total cells and migrated cells on the underside of the transwell membrane were detected by $\mathrm{H} 33342$ staining. Migration index was calculated as percentage of migrated cells related to total cells in the assay. Relative migration represents migration index compared to control cultures transfected with nonsense siRNA. Data are the means and SD from 3 experiments.

\section{Discussion}

Therapeutic approaches in children suffering from RMS are currently based on clinical determinants and histological findings. However, these prognostic and predictive factors may be improved with biological parameters for a better riskadapted treatment of these patients. Based on gene expression arrays, there is growing evidence that rhabdomyosarcoma is a molecular heterogeneous disease with multiple molecular subtypes.

Recently, array-based analysis of RMS samples described class prediction models with 10 genes to discriminate between RMA and RME (9). In the studies of Wachtel et al (6), Davicioni et al (7), and Lae et al (9) there were only very few common differentially expressed genes between RMA and RME. We made the same observation in our probe cohort (Table III). In the cited studies, comparisons were performed between RMS tissues with and without translocation or based on histological classification. The expression data of these 4 studies were obtained with the Affymetrix platform and has to be taken together to increase the number of samples and to apply a singular expression analysis algorithm in future. As a common unexpected finding from these studies, a gene encoding the cannabinoid receptor 1 (CNR1) was found highly up-regulated in RMA and therefore, the cannabinoid receptor agonists could represent a novel targeted approach for treatment of translocation-positive RMS. Inhibition of CNR1 with increased concentrations of AM28, an antagonistic inhibitor did not impair cell viability of the RMS cells ( $\mathrm{Rh} 30$ and $\mathrm{RD}$ ) at concentrations below $40 \mu \mathrm{M}$ (unpublished own data). However, agonists of CNR1 reduced RMS cell proliferation (23).

Similar to studies performed for breast cancer, considering patient data such as outcome, metastasis and drug response may facilitate discovery of gene-networks with prediction relevance for metastatic invasion and drug resistance in RMS. This implies the necessity for an increased number of primary RMS-samples, metastases and muscle tissue as reference. In our collective of 19 patients, the number of tissue samples was too low to build statistically relevant subgroups based on these parameters. Therefore, we focused on molecular networks characteristic for the main groups of RMA and RME.
Pathway analysis considers the already published interaction of gene products in different cellular systems and allows a focus on gene networks, which may be profoundly affected in tumor cells. Since the small number of tumor samples did not allow an unsupervised analysis of the gene expression with metastatic invasion as criterion, we depicted genes involved in metastatic invasion such as the transcription factors LMO4 and FOXF1 for functional analysis. These factors were over-expressed in RMA tissue samples. Comparison of pairs of original tumor samples and metastases may reveal other factors involved in the metastatic invasion of RMS.

LIM domain transcription factor LMO4 plays a role in breast cancer. Analysis of 177 primary invasive breast carcinomas revealed over-expression of LMO4 in proliferating mammary epithelium suggesting that deregulation of this gene may contribute to breast tumor genesis (24). Over-expression of LMO4 in non-invasive, immortalized human breast epithelial cells promoted cell motility and invasion (21). Our data revealed high expression levels in metastatic tumors and in the RMA cell line Rh30 with the highest invasion capability compared to the RD cell line. These findings overlap with the observations in breast cancer.

Genomic deletions of the forkhead box (FOX) gene family play an important role in the pathology of RMA as the major translocation involves FOXO1. FOXF1 is not linked in the cluster of FOXO1, however inactivating mutations of FOXF1 cause alveolar capillary dysplasia and other malformations (25). The low expression of FOXF1 in mice correlated with decreased expression of Vcam1, $\alpha-5$ integrin, Pdgf receptor- $\alpha$ and $\mathrm{Hgf}$, all of which are critical for cell adhesion and migration (22). Among them, we detected Vcam1, $\alpha-5$ integrin and Pdgf receptor- $\alpha$, on our arrays with a lower expression in RME than in RMA. However, they did not fulfill the selection criteria for the differential expression analysis. Low expression of FOXF1 in RME cells may correlate with reduced response capability of the RME cells to migration factors. A reduced invasion capability was expected for RD cells as LMO4 expression was low and FOX1 was almost undetectable. Consequently, a further lowering of FOXF1 expression in RD cells by siRNA did not contribute to the measured invasion. In concordance with the role of FOXF1 in the mouse embryonic development where the FOX gene cluster promotes mesenchymal cell differentiation, 
Table III. Comparative analysis of genes with differential expresion in RMS.

\begin{tabular}{lll}
\hline Davincioni et al vs. 534 & Lae et al vs. 534 & Wachtel et al vs. 534
\end{tabular}

MAGE-like 2

Extracellular matrix protein 1

Dual specificity phosphatase 4

MRNA; cDNA DKFZp762M127

POU domain, class 4, transcription factor 1

Bone morphogenetic protein 5

\section{Anaplastic lymphoma}

kinase (Ki-1)

Transcription factor 4

S100 calcium binding protein A10

Hairy and enhancer of split 1,

(Drosophila)

NEL-like 1 (chicken)

Transforming growth factor $\beta 1$ induced transcript 1

\section{Potassium intermediate/small} conductance calcium-activated channel, subfamily $N$, member 3

Fibrillin 2 (congenital contractural arachnodactyly)

E2F transcription factor 5, p130-binding

Polo-like kinase 2 (Drosophila)

Dual specificity phosphatase 4

Growth differentiation factor 8

Pleckstrin homology-like domain, family $\mathrm{A}$, member 1

EPH receptor A3

Low density lipoprotein receptor-related protein 5

Growth arrest-specific 2

Protein kinase, cAMP-dependent, regulatory, type II, $\beta$

3-hydroxy-3-methylglutarylcoenzyme A reductase
Adrenergic, $\alpha-2 \mathrm{~A}-$, receptor

Adrenergic, $\alpha-2 \mathrm{C}$-, receptor

Anaplastic lymphoma kinase (Ki-1)

Rho guanine nucleotide exchange factor (GEF) 4

Bone morphogenetic protein 5

Chromosome 14 open reading frame 132

CDC42 effector protein

(Rho GTPase binding) 4

Cannabinoid receptor 1 (brain)

Dystonin

Forkhead box F1

GA binding protein transcription factor, $\beta$ subunit 2

Potassium intermediate/small conductance calcium-activated channel, subfamily $\mathbf{N}$, member 3

Membrane-associated guanylate kinase, WW and PDZ domain containing 1

NEL-like 1 (chicken)

Oligodendrocyte lineage transcription factor 2

Paired-box gene 2

Pipecolic acid oxidase

Podocalyxin-like

Protein phosphatase $1 \mathrm{H}$

(PP2C domain containing)

Ryanodine receptor 3

Sodium channel, voltage-gated, type IV, $\alpha$

Solute carrier family 24

Transient receptor potential cation channel, subfamily $\mathrm{V}$, member 1

TSC22 domain family, member 2
Nuclear receptor subfamily 0 , group B, member 1

Potassium inwardly-rectifying channel, subfamily $\mathbf{J}$, member 5

Deleted in liver cancer 1

Cannabinoid receptor 1 (brain)

Homeobox C10

Fibrillin 2 (congenital contractural arachnodactyly)

RNA binding motif, single-stranded interacting protein 1

Pipecolic acid oxidase

Elastase 2A

Elastase 2B

Trefoil factor 3 (intestinal)

POU domain, class 4, transcription factor 1

Cholinergic receptor, nicotinic, $\beta 3$

Transcription factor AP-2 B

Listed are genes with differential expression in RMA vs. RME detected in previous studies and in this study. Genes in bold were detected in more than 2 studies. 
FOXF1 expression was higher in RMA compared to RME. It has to be assessed, whether restoring the FOXF1 expression in RME cells will be sufficient for a progression in the myogenic pathway differentiation (26).

So far no specific inhibitors of LMO4 and FOXF1 have been described. Therefore, modulating function of downstream targets of these transcription factors may be more attractive to design inhibitors of metastatic invasion in RMS $(27,28)$. Microarray technology is a powerful method not only to classify tumor samples, but also to select genes involved in crucial properties of cancer like proliferation, invasion and response to drugs. Our functional evaluation of LMO4 and FOXF1 opens up a molecular network to address metastatic invasion in RMS.

\section{Acknowledgements}

Parts of this work were funded by the Fortüne program of the Medical Faculty of the University of Tübingen (1432-0-0). The authors wish to thank Karl-Ludwig Schäfer, $\mathrm{PhD}$ for providing tumor samples and, Michael Neubauer from the Department of Gynaecology and Obstetrics, University of Tübingen, Germany, for his advice with migration/invasion assay.

\section{References}

1. McDowell HP: Update on childhood rhabdomyosarcoma. Arch Dis Child 88: 354-357, 2003.

2. Dantonello TM, Int-Veen C, Harms D, et al: Cooperative trial CWS-91 for localized soft tissue sarcoma in children, adolescents, and young adults. J Clin Oncol 27: 1446-1455, 2009.

3. Hayes-Jordan A and Andrassy R: Rhabdomyosarcoma in children. Curr Opin Pediatr 21: 373-378, 2009.

4. Grass B, Wachtel M, Behnke S, Leuschner I, Niggli FK and Schafer BW: Immunohistochemical detection of EGFR, fibrillin-2, P-cadherin and AP2beta as biomarkers for rhabdomyosarcoma diagnostics. Histopathology 54: 873-879, 2009.

5. Parham DM, Qualman SJ, Teot L, et al: Correlation between histology and PAX/FKHR fusion status in alveolar rhabdomyosarcoma: a report from the Children's Oncology Group. Am J Surg Pathol 31: 895-901, 2007.

6. Wachtel M, Dettling M, Koscielniak E, et al: Gene expression signatures identify rhabdomyosarcoma subtypes and detect a novel $\mathrm{t}(2 ; 2)(\mathrm{q} 35 ; \mathrm{p} 23)$ translocation fusing PAX3 to NCOA1. Cancer Res 64: 5539-5545, 2004.

7. Davicioni E, Anderson MJ, Finckenstein FG, et al: Molecular classification of rhabdomyosarcoma-genotypic and phenotypic determinants of diagnosis: a report from the Children's Oncology Group. Am J Pathol 174: 550-564, 2009.

8. Goldstein M, Meller I, Issakov J and Orr-Urtreger A: Novel genes implicated in embryonal, alveolar, and pleomorphic rhabdomyosarcoma: a cytogenetic and molecular analysis of primary tumors. Neoplasia 8: 332-343, 2006.

9. Lae M, Ahn EH, Mercado GE, et al: Global gene expression profiling of PAX-FKHR fusion-positive alveolar and PAX-FKHR fusion-negative embryonal rhabdomyosarcomas. J Pathol 212: 143-151, 2007.

10. Schaaf GJ, Ruijter JM, van RF, et al: Full transcriptome analysis of rhabdomyosarcoma, normal, and fetal skeletal muscle: statistical comparison of multiple SAGE libraries. FASEB J 19: 404-406, 2005.
11. Davicioni E, Anderson JR, Buckley JD, Meyer WH and Triche TJ: Gene expression profiling for survival prediction in pediatric rhadomyosarcomas: a report from the Children's Oncology Group 3. J Clin Oncol 28: 1240-1246, 2010.

12. Mercado GE, Xia SJ, Zhang C, et al: Identification of PAX3FKHR-regulated genes differentially expressed between alveolar and embryonal rhabdomyosarcoma: focus on MYCN as a biologically relevant target 1 . Genes Chromosomes Cancer 47: 510-520, 2008.

13. Romualdi C, De PC, Tombolan L, Bortoluzzi S, Sartori F, Rosolen A and Lanfranchi G: Defining the gene expression signature of rhabdomyosarcoma by meta-analysis 1 . BMC Genomics 7: 287, 2006.

14. Amstutz R, Wachtel M, Troxler H, et al: Phosphorylation regulates transcriptional activity of PAX3/FKHR and reveals novel therapeutic possibilities. Cancer Res 68: 3767-3776, 2008.

15. Ebauer M, Wachtel M, Niggli FK and Schafer BW: Comparative expression profiling identifies an in vivo target gene signature with TFAP2B as a mediator of the survival function of PAX3/ FKHR. Oncogene 26: 7267-7281, 2007.

16. Armistead PM, Salganick J, Roh JS, et al: Expression of receptor tyrosine kinases and apoptotic molecules in rhabdomyosarcoma: correlation with overall survival in 105 patients. Cancer 110: 2293-2303, 2007

17. Cen L, Arnoczky KJ, Hsieh FC, et al: Phosphorylation profiles of protein kinases in alveolar and embryonal rhabdomyosarcoma. Mod Pathol 20: 936-946, 2007.

18. Tordai A, Liedtke C and Pusztai L: Metastatic gene signatures and emerging novel prognostic tests in the management of early stage breast cancer. Clin Exp Metastasis 26: 625-632, 2009.

19. Kapushesky M, Kemmeren P, Culhane AC, et al: Expression Profiler: next generation - an online platform for analysis of microarray data. Nucleic Acids Res 32: W465-W470, 2004.

20. Habig K, Walter M, Poths S, Riess O and Bonin M: RNA interference of LRRK2-microarray expression analysis of a Parkinson's disease key player. Neurogenetics 9: 83-94, 2008.

21. Sum EY, Segara D, Duscio B, et al: Overexpression of LMO4 induces mammary hyperplasia, promotes cell invasion, and is a predictor of poor outcome in breast cancer. Proc Natl Acad Sci USA 102: 7659-7664, 2005.

22. Kalinichenko VV, Zhou Y, Bhattacharyya D, Kim W, Shin B, Bambal K and Costa RH: Haploinsufficiency of the mouse Forkhead Box f1 gene causes defects in gall bladder development. J Biol Chem 277: 12369-12374, 2002.

23. Oesch $\mathrm{S}$, Walter $\mathrm{D}$, Wachtel $\mathrm{M}$, et al: Cannabinoid receptor 1 is a potential drug target for treatment of translocation-positive rhabdomyosarcoma. Mol Cancer Ther 8: 1838-1845, 2009.

24. Visvader JE, Venter D, Hahm K, et al: The LIM domain gene LMO4 inhibits differentiation of mammary epithelial cells in vitro and is overexpressed in breast cancer. Proc Natl Acad Sci USA 98: 14452-14457, 2001.

25. Stankiewicz P, Sen P, Bhatt SS, et al: Genomic and genic deletions of the FOX gene cluster on 16q24.1 and inactivating mutations of FOXF1 cause alveolar capillary dysplasia and other malformations. Am J Hum Genet 84: 780-791, 2009.

26. Wijnaendts LC, van der Linden JC, van Unnik AJ, Delemarre JF, Barbet JP, Butler-Browne GS and Meijer CJ: Expression of developmentally regulated muscle proteins in rhabdomyosarcomas. Am J Pathol 145: 895-901, 1994.

27. Montanez-Wiscovich ME, Seachrist DD, Landis MD, Visvader J, Andersen B and Keri RA: LMO4 is an essential mediator of ErbB2/HER2/Neu-induced breast cancer cell cycle progression. Oncogene 28: 3608-3618, 2009.

28. Wang N, Lin KK, Lu Z, et al: The LIM-only factor LMO4 regulates expression of the BMP7 gene through an HDAC2dependent mechanism, and controls cell proliferation and apoptosis of mammary epithelial cells. Oncogene 26: 6431-6441, 2007. 